\title{
Integrative analysis of cutaneous skin tumours using ultrasonogaphic criteria. Preliminary results.
}

\author{
Diana Crișan', Alexandru Florin Badea², Maria Crișan", Ioana Rastian¹, Laura Gheuca \\ Solovastru ${ }^{3}$, Radu Badea ${ }^{4}$
}

${ }^{1}$ Dermatology Department, Clinical Municipal Hospital, Cluj-Napoca, ${ }^{2}$ Department of Maxilofacial Surgery "Iuliu Hatieganu" University of Medicine and Pharmacy, Cluj-Napoca, ${ }^{3}$ Dermatology Department, "Gr. T. Popa" University of Medicine and Pharmacy, Iasi, ${ }^{4}$ Department of Ultrasonography, "O. Fodor" Institute of Gastroenterology and Hepatology, ”Iuliu Hatieganu” University of Medicine and Pharmacy, Cluj-Napoca, Romania

All authors have equal contributions to this work

\begin{abstract}
The aim of this study is to identify the US features of skin tumors, especially morphological and vascular, in order to develop an integrative and differentiating imaging model for benign and malignant skin tumors. Material and method: Twenty three patients with solid skin tumors were included in the study. The diagnostic procedures were clinical examination, dermoscopy, multimodal ultrasonography (US), using high frequency and conventional US, contact elastography, and i.v. contrast enhanced ultrasound (CEUS). Results: The US characteristics of the basal cell carcinomas were: hypoechoic, inhomogeneous masses, with hyperechoic or anechoic areas, depending on the histological differentiation, increased rigidity, uneven vascularization at Doppler examination, central or mixed type circulatory model, with 1-2 supply vessels, velocity $>2$ $\mathrm{cm} / \mathrm{s}$, intensely inhomogeneous load of the contrast agent ( CA) and quick wash out time. The benign tumors were hypoechoic or echoic masses, with inhomogeneous structure, Doppler signal present only in dermofibromas, peripheral circulation model, velocities $<2.00 \mathrm{~cm} / \mathrm{s}$, a weak and uneven loading of the CA in the vascular bed, and a slow wash out time. Analysis of the CA dynamics evidenced a significantly higher value for the wash out time in the malignant tumors (38.2s \pm 15.15$)$ as compared to the benign ones $(54.2 \mathrm{~s} \pm 8.5)$. Particularly the tumor thickness examination by HFUS evidences an ultrasound index that may be considered as a statistically significant predictive factor $(\mathrm{p}<0.05)$, highly sensitive $(\mathrm{r}=0.97)$ for the non-invasive assessment of the histological Breslow index. Elastography did not represent a differentiation examination in the cases studied. Conclusions: Ultrasound allows a complex, multimodal approach of skin tumors, which completes clinical and histological examinations, orients the therapeutic management and may assessthe therapeutic efficacy and the tumoral prognosis.
\end{abstract}

Keywords: skin, skin tumors, ultrasonography, elastography, contrast agent

\section{Introduction}

Basal cell carcinomas (BCC) are the most frequent malignant skin tumors. Considered to be characteristic for the age over 40 years, their incidence is growing in

Received 24.06.2014 Accepted 21.07.2014

Med Ultrason

2014, Vol. 16, No 4, 285-290

Corresponding author: Prof. Dr. Maria Crisan Dermatology Clinic, "Iuliu Hatieganu"

University of Medicine and Pharmacy

3-5 Clinicilor Street

400359 Cluj-Napoca, Romania

Phone:0040744766496

Email: maria.crisan@umfcluj.ro the younger population, probably due to uncontrolled exposure to ultraviolet rays [1]. The early diagnosis and minimally invasive therapy that preserves the skin aspect and function is a constant preoccupation [2]. The clinical diagnosis based on several criteria (aspect, evolution time, location) was initially completed by dermoscopy, which evidences the intratumoral vascular network, and lately by conventional and high frequency ultrasonography (US). US examination provides additional information that completes the clinical and pathological diagnosis. It is easily accepted by the patient being a noninvasive and inexpensive imaging technique, which provides useful information for the management, evolution, and prognosis of the skin tumors [3]. US examination has 
gained ground in dermatology due to modern technologies and targets, beside tumoral pathologies, also chronic inflammatory diseases, the efficiency of topic and general therapies, including the antiaging therapies etc. [4]. The so called "conventional" US techniques provide morphological data in sectional formats (bidimensional or 2D US) and vascular data (quantitative Doppler US - pulse mode, or qualitative - color flow map mode). In the last years new US techniques were introduced, such as elastography, contrast enhanced ultrasound (CEUS) [5], and high-frequency ultrasound (HFUS) [6]. Each technique provides particular information, which may be integrated into the overall US examination to achieve a complex assessment of the nature of the tumors, orienting the management toward an optimal and minimally invasive therapy, without risks of local recurrence.

The aim of this study is to identify the US features of skin tumors, especially morphological and vascular, in order to develop an integrative and differentiating imaging model for benign and malignant skin tumors.

\section{Material and method}

This prospective linear study comprised 23 patients, 15 men and 8 women, aged between 23 and 83 years. All the patients presented nodular tumors of the skin, on photo-exposed or photo-protected areas. The patients were identified in the Dermatology Clinic outpatient unit and submitted to ultrasound examinations at the $3^{\text {rd }}$ Medical Clinic between October 2012 - 10 May 2013.

The inclusion criteria were the small to medium sized nodular tumors, without marked ulcerations, situated at skin level (face, extremities, or back). All the patients gave their informed consent for the study, which was approved by the Ethics Committee of the "Iuliu Hatieganu" University of Medicine and Pharmacy.

The patients studies were submitted to the same protocol: clinical and dermatoscopic examinations, using a HEINE 20 device in water drop, high frequency and conventional US, performed according to standard procedures [7-9], and surgical excision. In all the cases the diagnosis was confirmed by histology. Ultrasound results were compared with that of histological examination.

HFUS with a Dermascan $20 \mathrm{MHz}$ device (Cortex Technologies Denmark) assessed the depth of the tumor in $\mathrm{mm}$ from the epidermal level. The US tumoral depth (US index) was compared with Breslow's index. The clinical diagnosis associated with dermoscopy evidenced the elements of malignancy (superficial, dilated, branched vessels, situated at the center or the periphery of tumors) and the benign signs (no visible vessels, or few thin vessels in radial display at the tumor periphery in the case of dermofibromas)

Conventional US (2D) was performed using the last generation device iU22, Phillips, The Netherlands, with a soft tissue transducer of 7-13 MHz frequency. For each lesion the echogenicity (in relation to normal dermal tissue), the homogeneity, and the tumoral depth were appreciated. Tumoral rigidity was assessed by color touch elastography. Conventionally, blue means a rigid tissue, red elastic tissue, and green intermediate rigidity.

Macro-circulation at the level of the tumoral bed was assessed by color flow map (CFM) Doppler - for evidencing vessels, and pulse Doppler - for differentiating between veins and arteries and for measuring velocity. The CFM model was labeled organized (branching vessels) or disorganized (dislodged, interrupted vessels), with a central, peripheral, or mixed distribution, according to the location of the vessels in the tumor. The arterial or venous nature was assessed based on the flow type (continuous or systolic-diastolic). Velocities were measured by placing the Doppler sample in the vessel center, using an adequate correction angle.

CEUS used a standard technique [10] and assessed microcirculation in the tumoral bed. The contrast agent (CA) (16 ccm SonoVue, Bracco, Italy), prepared on the spot, was injected i.v. at the elbow level, followed by 10 $\mathrm{ccm}$ saline. In all the cases dual examination was performed, the US data being displayed simultaneously on the screen. The examination was continuous after the injection time and lasted about 2 minutes. The CEUS allowed the assessment of vascular dynamics, based on the following parameters: the time to tumor uptake (seconds after i.v. injection), time from the injection of the CA until the maximum intensity is reached (time to peak), time to wash out (from the peak to the disappearance of the CA) and total CA duration (between loading and total disappearance). The disposition of the intratumoral vessels and the CA penetration model in the arterial time defined a uniform loading model (complete load of the vascular bed), or an uneven loading model.

The histological examination was performed using hematoxilin eosin staining. The histological diagnosis established the type of histological differentiation and the in-depth extension (Breslow index), measured from the epidermal granulous layer to the deepest tumoral point.

A comparative analysis of the data targeted only the relevant parameters for the tumoral description. We performed a correlation analysis (Pearson's coefficient), a regression analysis (simple linear regression) and a graphic representation between the ultrasound and histological findings. The correlation between the vascular 
times of the CA dynamics was graphical (by observation) and by calculating averages. The interpretation of the vascular times was only graphical, due to the small number of cases in the benign tumor group.

\section{Results}

There were 23 tumors evidenced in our study, 18 malignant and 5 benign (Table I). The ultrasound findings on the tumors are summarized in table II. Thus, the malignant tumors were hypoechoic masses, inhomogeneous, with abundant, disorganized arterial-type vessels disposed at the center and the periphery, circulation velocity $>2 \mathrm{~cm} / \mathrm{sec}$, intensely inhomogeneous load of the $\mathrm{CA}$ and quick wash out time than in benign tumors. The benign tumors were hypoechoic or echoic masses, with an inhomogeneous structure, Doppler signal present only in dermofibromas, peripheral circulation model, tributary to the visualized supply vessels, velocities $<2.00 \mathrm{~cm} / \mathrm{sec}$. CEUS evidenced a weak and uneven loading of the CA in the vascular bed, the presence of supply vessels in the 5 benign tumors, and a slow wash-out time (fig 1, fig 2). Regarding the tumor thickness, there was a significant correlation coefficient of 0.97 between Breslow's index and the HFUS values (fig 3). The regression analysis indicates that the histological index may be assessed using the US index. Therefore the US index may be considered a statistically significant predictive factor $(\mathrm{p}<0.05)$, highly accurate $(\mathrm{r}=-0.97)$ in the non-invasive assessment of the histological index. Therefore, the mean theoretical (expected) value of the histological index will be 2.26 $\mathrm{mm}$ at a US index of $2.7 \mathrm{~mm}$

Also, a significant differentiation appears to be between the wash out times of the different types of lesions. The analysis of the CA dynamics evidenced a significantly higher value for the wash out time in the malignant tumors $(38.2 \mathrm{~s} \pm 15.15)$ as compared to the benign ones (54.2s \pm 8.5$)$ (fig.4). In our study color touch elastography evidenced rigidity in all the lesions studied.

Tabel I. Histological diagnosis of the skin tumors

\begin{tabular}{ll}
\hline Histological diagnosis \\
\hline Malignant tumors $(\mathrm{n}=18)$ \\
Solid 4 \\
Keratotic differentiation 6 \\
Adenoid/cystic differentiation 7 \\
Metatypical 1 \\
Benign tumors $(\mathrm{n}=5)$ \\
Keratoacanthoma 1 \\
Hypertrophic actinic keratosis 2 \\
Dermofibroma 2 \\
\hline
\end{tabular}

$n=$ number of cases

Tabel II. Comparative findings in malignant and benign skin tumors

\begin{tabular}{lll}
\hline Criteria & Malignant tumors (n=18) & Benign tumors (n=5) \\
\hline $\begin{array}{l}\text { 1. Morphometry } \\
\text { Echogenicity }\end{array}$ & Hypoechoic $(18 / 18)$ & Hypoechoic $(2 / 5)$ \\
& & Echoic $(3 / 5)$ \\
Echo-structure & Inhomogeneous $(18 / 18)$ & Inhomogeneous $(5 / 5)$ \\
Tumoral depth & 2D US: $1.5-6 \mathrm{~cm}$. & 2D US: $0.8-1.2 \mathrm{~cm}$. \\
& Dermascan: $1-6.02 \mathrm{~mm}$. & Dermascan: $1-1.05 \mathrm{~mm}$. \\
2. Tumoral macro-circulation (Doppler US) & & \\
Doppler signal & Present $16 / 18$ & Absent $3 / 5$ \\
Evidence of supply vessels & $15 / 18$ & $2 / 5$ \\
Circulatory pattern & Disorganized & Relatively organized \\
Vessel distribution & Central $12 / 18$ & Peripheral $5 / 5$ \\
& Mixed $6 / 18$ & $<2 \mathrm{~cm} / \mathrm{sec}$ \\
Velocity & $>2 \mathrm{~cm} / \mathrm{sec}$ & \\
3. Tumoral microcirculation (CEUS) & & Weak, inhomogeneous $5 / 5$ \\
CA uptake pattern & Inhomogeneous $18 / 18$ & Supply vessels $5 / 5$ \\
& Supply vessels $18 / 18$ & $10-20$ seconds \\
CA uptake & $9-22$ seconds & $14-26$ seconds \\
Time to the maximum intensity of the CA signal & $12-33$ seconds & $27-38$ seconds \\
Complete wash out (since peak) & $25-90$ seconds & $78-88$ seconds \\
Persistence of signal & $60-100$ seconds & \\
4. Elastography & & Relevantly rigid $5 / 5$ \\
Rigidity & Relevantly rigid $18 / 18$ &
\end{tabular}

$\mathrm{n}=$ number of cases 


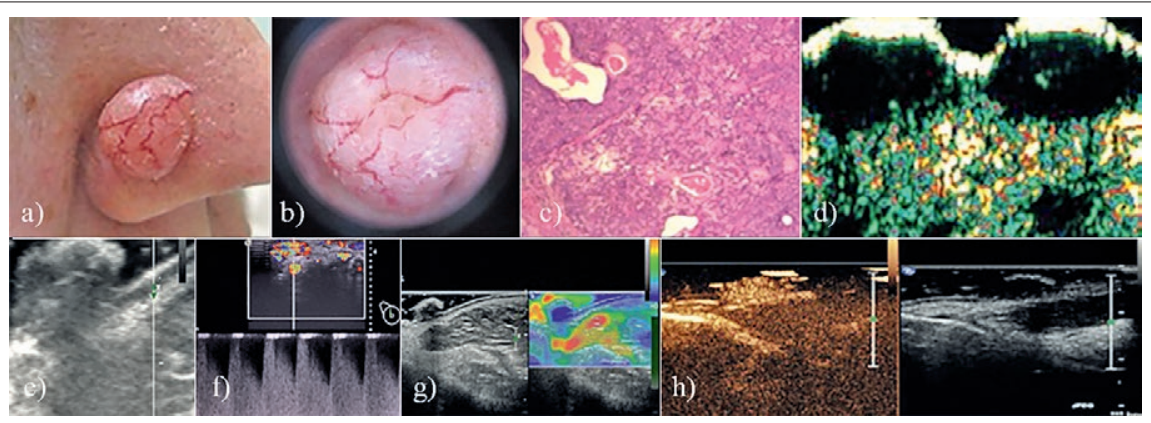

Fig 1. Nodular basal cell carcinoma: a) clinical aspect; b) dermoscopic aspect: branched, dilated tumoral vessels; c) histology: clusters of basaloid cells with keratotic and cystic differentiation, infiltrating the dermis; d) HFUS: hypoechoic masses, with hyperechoic echoes corresponding to keratotic pearls; e) 2D US: hypoechoic, inhomogeneous structure, blurred delimitation, shadow cone, the expression of intratumoral keratin masses; f) Doppler examination: the exophytic tumor is evidenced; Doppler image reveals arterial blood flow type, with high velocity and turbulences; g) elastography: relevantly rigid mass; $h$ ) CEUS dynamics: weak inhomogeneous load of the vascular bed, one supply vessel, peak load (24 sec).

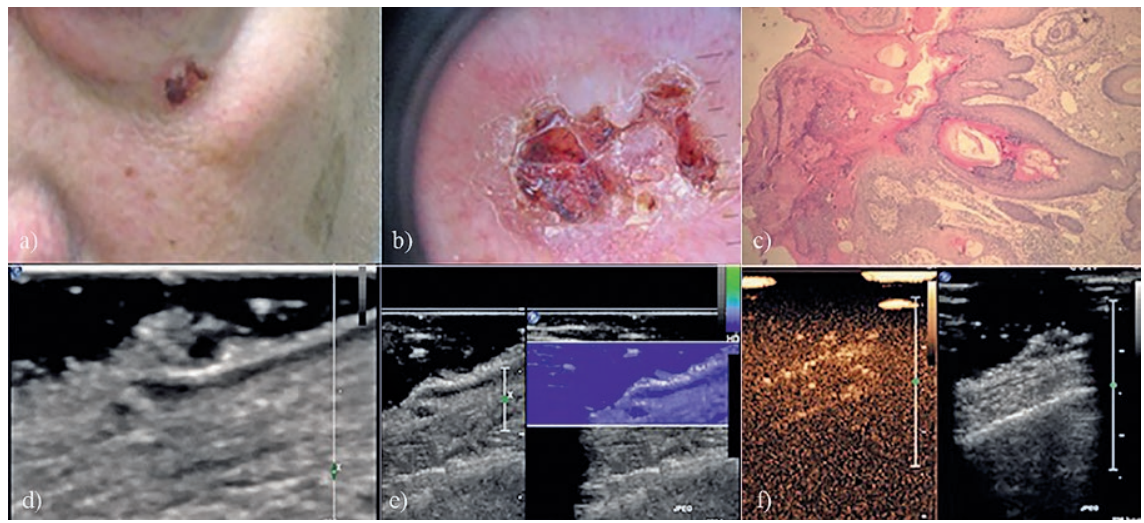

Fig 2. Actinic keratosis: a) clinical aspect; b) dermoscopy aspect: superficial ulceration, keratin deposits, no intratumoral vessels visualized; c) histology: keratin deposits, papillomatosis, inflammatory infiltrate; d) 2D US: hypoechoic mass, unclear contour, absent Doppler signal, no supply vessel; e) elastography: rigid tumor; f) CEUS dynamics; weak, inhomogeneous loading, supply vessel, peak load $(26 \mathrm{sec})$.

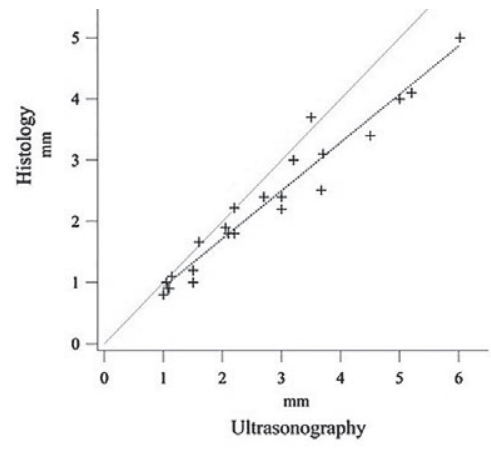

Fig 3. Relationship between the histological and ultrasound measurements. The ensuing correlation coefficient was 0.97 .

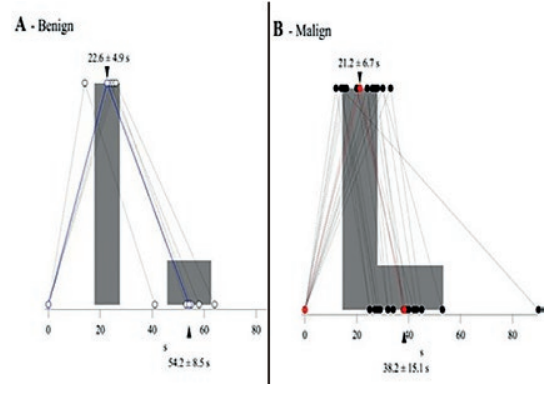

Fig 4. Dynamics of circulation in the benign and malignant skin tumors. Time of uptake and wash-out of the benign (A) and malignant (B) tumors. Circles with dotted lines represent individual values. Colored circles and lines (blue for benign, red for malignant tumors) represent average durations. The grey squares represent $\pm \mathrm{SD}$ values around the mean ones. Mean \pm SD values are also indicated below arrows, for clarity. 


\section{Discussions}

Though the role of US in the study of skin tumors has made the object of many articles, there are sufficient additional aspects, which may provide valuable information to complete the clinical, dermoscopic and histological data $[11,12]$. US examination is a valuable method of investigation of the skin tumors in correlation with the clinical and pathological examinations. It is accessible, easily accepted by the patient, providing highly sensitive information, but reduced in specificity [13]. The various US equipments, with frequencies ranging between 7-100 $\mathrm{MHz}$ used in cutaneous investigations were studied and revealed the advantages and drawbacks of US in dermatology $[13,14]$.

Our results are similar with the results of Wells et al [15] that shows a significant correlation between US and the histological findings regarding tumor thickness. The small differences, due to histological or US overestimation, may be explained by the presence of the peri-lesional tumoral infiltrate, the retraction of tissue induced by the histological section, and, not least, the examiner's experience, US being an operator-dependent method [16-18]. Therefore, HFUS may be implemented as a non-invasive complementary method of assessment of tumoral depth, similar in precision to histology, which is important for the therapeutic management.

Our study shows the need for the cumulative use of ultrasonographic criteria focused on the morphology and the haemodynamics of solid skin tumors. In our cases a correlation between the US aspect and histology, which is relevant for the sensitivity, was observed. The inhomogeneous structures (hyperechoic or anechoic) were correlated with rich intratumoral vascularization, fibrous stroma, type of histological differentiation (keratotic, adenoid, or cystic). Similar aspects, but without mentioning the correlation with the histological differentiation, have been reported in literature [19].

The circulatory model provides information that may contribute to the consolidation of the clinical diagnosis. Thus, the Doppler circulation pattern, central or mixed, with higher velocities in the malignant tumors $(>2 \mathrm{~cm} /$ $\mathrm{sec}$ ), in comparison with the peripheral pattern with lower velocities in the benign tumors $(<2 \mathrm{~cm} / \mathrm{sec})$ are elements that correlate with the clinical and histological findings. Similar data were reported in literature $[19,20]$. As compared to capillary velocities in physiological conditions $(1 \mathrm{~mm} / \mathrm{sec})$, we consider that our findings are relevant for tumoral lesions. The lack of evidence of supply vessels and the vascular bed in certain cases reveal the limitations of the Doppler examination, which are overcome by CEUS [21].
The analysis of the CA upload suggest that the tumoral vascular dynamics depends on several factors (vascular resistance, shunts, histological type, location). We did not find reports in literature regarding the $\mathrm{CA}$ vascular dynamics in BCC. Studies in a larger number of patients are necessary to establish a circulation pattern of skin tumors. The CA tumor uptake time and the time to the maximum intensity of the signal (time to peak) was not relevant in our study. According to our results the wash out time could represent an important criterion of differentiation between skin tumors. An analytical study in larger groups would be required to establish the wash-out time as an objective criterion in the differentiation of skin tumors. CEUS is a frequently used method in the assessment of the tumoral pathology at the level of the internal organs, but as we show here, can also be successfully used in the field of oncodermatology [22-24].

Special attention should be paid to the tumoral rigidity. In our study color touch elastography evidenced rigidity in all the lesions studied. According to the literature elastography has a sensitivity $>95 \%$ and a specificity of $85 \%$ in evidencing and classifying extra-cutaneous tumors (breast, prostate) [25]. The role of elastography in skin tumors is scarcely debated in literature. Some authors have postulated the role of elastography in melanocytic tumors and skin carcinomas, as well as its high predictive value in patients with clinically node-negative skin cancer. [26,27]. Additional studies are necessary to make elastography an important imaging marker for the differentiation of skin tumors.

Our study revealed the necessity of using the imaging criteria focused on the morphological and hemodynamic aspects of skin tumors in a cumulative manner. The complex, multimodal approach, correlated with the clinical and histological findings represents a non-invasive excellent method of differentiation of skin tumors. According to our findings, the imaging malignancy criteria in $\mathrm{BCC}$ are: inhomogeneous structure, rigidity, abundant, inhomeogeneous, arterial vascularization with velocities $>2$ $\mathrm{cm} / \mathrm{sec}$, vessels located at the center and periphery, intense and irregular load of the contrast agent, quick wash out time.

The limitations of the study are related to the small patient number, the group conveniently selected, and the operator-dependent investigation, which might be responsible for the errors occurring in the results.

In conclusion, we may state that US is a method of virtual biopsy. It allows a complex, multimodal approach of skin tumors, which completes clinical and histological examinations, orients the therapeutic management and assesses the therapeutic efficacy and the tumoral prognosis. It represents a modern, non-invasive technique of 
translational diagnosis from the clinical field to the field of fundamental research.

Acknowledgements: We hereby thank Prof. Dr. F. Amzica, for his assistance in preparing the graphics.

Conflict of interest: none

\section{References}

1. Bolognia JL, Jorizzo JL, Rapini RP. Dermatology. $1^{\text {st }}$ ed. Madrid: Elsevier Mosby; 2003.

2. Saurat JH, Lachapelle JM, Lipsker D, Thomas L. Dermatologie et infections sexuellement transmissible. $5^{\text {th }}$ ed. Elseier-Masson; 2009.

3. Crisan M, Crisan D, Sannino G, Lupsor M, Badea R, Amzica F. Ultrasonographic staging of cutaneous malignant tumors: an ultrasonographic depth index. Arch Dermatol Res 2013; 305: 305-313.

4. Crisan M, Badea R, Lupsor M, Moldovan M, Crisan D, Boca A. Imagistic evaluation of the efficacy of anti-ageing and hydrating products on the cutaneous ageing process. Preliminary data. Farmacia in press

5. Kleinerman R, Whang TB, Bard RL, Marmur ES. Ultrasound in dermatology: Principles and applications. J Am Acad Dermatol 2012; 67: 478-487.

6. Korde VR, Bonnema GT, Xu W, et al. () Using optical coherence tomography to evaluate skin sun damage and precancer. Lasers Surg Med 2007; 39: 687-695.

7. Badea R, Crisan M, Lupsor M, Fodor L. Diagnosis and characterisation of cutaneous tumors using combined ultrasonographic procedures (conventional and high resolution ultrasonography). Med Ultrason 2010; 12: 317-322.

8. Crisan M, Cattani C, Badea R, et al. Computational Science and its Applicatios -ICCSA 2010. International Conference Fukoka, Japan, March 23-26, 2010. Proceedings, Part II Vol. 6017, 1st Edition. 2010; XIX: 602.

9. Cammarota T, Pinto F, Magliaro A, Sarno A. Current uses of diagnostic high-frequency US in dermatology. Eur J Radiol 1998; 27 Suppl 2: S215-S223.

10. Piscaglia F, Nolsoe C, Dietrich CF, et al. The EFSUMB Guidelines and Recommendations on the Clinical Practice of Contrast Enhanced Ultrasound (CEUS): update 2011 on non-hepatic applications. Ultraschall Med 2012; 33: 33-59.

11. Vogt M, Knuttel A, Hoffmann K, Altmeyer P, Ermert H.( Comparison of high frequency ultrasound and optical coherence tomography as modalities for high resolution and non invasive skin imaging. Biomed Tech (Berl) 2003; 48: 116-121.

12. Badea AF, Tamas-Szora A, Clichici S, et al. Contrast enhanced ultrasonography (CEUS) in the characterization of tumor microcirculation. Validation of the procedure in the animal experimental model. Medical ultrasonography 2013; 15: 85-94.

13. Bamber J, Cosgrove D, Dietrich CF, et al. EFSUMB guidelines and recommendations on the clinical use of ultrasound elastography. Part 1: Basic principles and technology. U1traschall Med 2013; 34: 169-184.

14. Lutz HT, Soldner R. Basic physics of ultrasound. In: Lutz $\mathrm{H}$, Buscarini E (eds.). Manual of diagnostic ultrasound, (2nd Ed.). Vol. 1. World Health Organisation. Gutenberg Press Ltd, Malta 2011: 1-4.

15. Wells PN, Liang HD. Medical ultrasound: imaging of soft tissue strain and elasticity. J R Soc Interface 2011;8:15211549.

16. Fornage BD, McGavran MH, Duvic M, Waldron CA. Imaging of the skin with 20-MHz US. Radiology 1993;189:6976.

17. Guitera P, Li LX, Crotty K, et al. Melanoma histological Breslow thickness by $75-\mathrm{MHz}$ ultrasonography. Br J Dermatol 2008; 159: 364-369.

18. Dudea SM, Lenghel M, Botar-Jid C, Vasilescu D, Duma M. Ultrasonography of superficial lymph nodes:benign vs. malignant. Med Ultrason 2012; 14: 294-306.

19. Wortsman X, Jemec G. Dermatologic Ultrasound with Clinical and Histological Correlations. New York:Springer; 2013.

20. Kohl E, Steinbauer J, Landthaler M, Szeimies RM. Skin ageing. J Eur Acad Dermatol Venereol 2011; 25: 873-884.

21. Badea R, Crisan M, Lupsor M, Fodor L. Diagnosis and characterisation of cutaneous tumors using combined ultrasonographic procedures (conventional and high resolution ultrasonography). MedUtrason 2010; 12: 317-322.

22. Claudon M, Cosgrove D, Albrecht T, et al. Guidelines and good clinical practice recommendations for contrast enhanced ultrasound (CEUS) - update 2008. Ultraschall in Med 2008; 29: 28-44.

23. Seitz K, Bernatik T, Strobel D, et al. Contrast-enhanced ultrasound (CEUS) for the characterization of focal liver lesions in clinical practice (DEGUM Multicenter Trial): CEUS vs. MRI - a prospective comparison in 269 patients. Ultraschall in Med 2010; 31: 492-499.

24. Strobel D, Seitz K, BlankW, et al. Contrast-enhanced ultrasound for the characterization of focal liver lesions - diagnostic accuracy in clinical practice (DEGUM multicenter trial). Ultraschall in Med 2008; 29: 499-505.

25. Ginat DT, Destonis SV, Barr RG, Castaneda B, Strang JG, Rubens DJ. US elastography of breast and prostate lesions. Radiographics 2009; 29: 2007-2016.

26. Aoyagy S, Izumi K, Hata H, Kawasaki H, Shimizu H. Usefulness of real-time elastography for detecting lymph-node metastases in squamous cell carcinoma. Clin Exp Dermatol 2009; 34: e744-e777.

27. Schmidt-Wendtner MH, Dill-Muller D. Ultrasound technology in dermatology. Semin Cutan Med Surg 2008; 27 : 44-51. 